\title{
Hyers-Ulam stability and applications in gauge spaces
}

Monica Bota, T. Petra Petru, and G. Petruşel 


\title{
HYERS-ULAM STABILITY AND APPLICATIONS IN GAUGE SPACES
}

\author{
M. BOTA, T. P. PETRU, AND G. PETRUŞEL
}

Received 18 September, 2012

\begin{abstract}
Using the weakly Picard operator technique, we will present some Ulam- Hyers stability results for operatorial equations and some applications in gauge spaces.

2000 Mathematics Subject Classification: 47H10; 54H25

Keywords: Hyers-Ulam stability, weakly Picard operator, $\psi$-weakly Picard operator, fixed point, integral equation
\end{abstract}

\section{INTRODUCTION}

In 1959, G. Marinescu [10] extended the Banach Contraction Principle to locally convex spaces, while I. Colojoară [4] and N. Gheorghiu [7] to gauge spaces and R. J. Knill [9] to uniform spaces. In 1971, Cain and Nashed [3] extended the notion of contraction to Hausdorff locally convex linear spaces. They showed that on sequentially complete subset, the Banach Contraction Principle is still valid. V.G. Angelov [1] introduced the notion of generalized $\varphi$-contractive single-valued map in gauge spaces in 1987, meanwhile the concept for multivalued operators was given in 1998 (see V.G. Angelov [2]). In 2000, M. Frigon [6] introduced the notion of generalized contraction in gauge spaces and proved that every generalized contraction on a complete gauge space (sequentially complete gauge space) has a unique fixed point.

Definition 1. Let $X$ be any set. A map $p: X \times X \rightarrow \mathbb{R}_{+}$is called a pseudometric (or, a gauge) in $X$ whenever

(1) $p(x, y) \geq 0$, for all $x, y \in X$;

(2) If $x=y$, then $p(x, y)=0$;

(3) $p(x, y)=p(y, x)$, for all $x, y \in X$;

(4) $p(x, z) \leq p(x, y)+p(y, z)$, for every triple of point.

This work was possible with the financial support of the Faculty of Business, Babeş-Bolyai University Cluj-Napoca. 
Definition 2. A family $\mathcal{P}=\left\{p_{\alpha}\right\}_{\alpha \in A}$ of pseudometrics on $X$ (or a gauge structure on $X$ ), where $A$ is a directed set, is said to be separating if for each pair of points $x, y \in X$, with $x \neq y$, there is a $p_{\alpha} \in \mathcal{P}$ such that $p_{\alpha}(x, y) \neq 0$.

A pair $(X, \mathcal{P})$ of a nonempty set $X$ and a separating gauge structure $\mathcal{P}$ on $X$ is called a gauge space.

It is well known (see Dugundji [5], pages 198-204) that any family $\mathcal{P}$ of pseudometrics on a set $X$ induces on $X$ a uniform structure $\mathcal{U}$ and conversely, any uniform structure $\mathcal{U}$ on $X$ is induced by a family of pseudometrics on $X$. In addition, we have that $\mathcal{U}$ is separating (or Hausdorff) if and only if $\mathcal{P}$ is separating. Thus we may identify the gauge spaces and the Hausdorff uniform spaces.

A sequence $\left(x_{n}\right)_{n \in \mathbb{N}}$ of elements in $X$ is said to be Cauchy if for every $\varepsilon>0$ and $\alpha \in A$, there is an $N$ with $p_{\alpha}\left(x_{n}, x_{n+p}\right) \leq \varepsilon$ for all $n \geq N$ and $p \in \mathbb{N}$.

The sequence $\left(x_{n}\right)_{n \in \mathbb{N}}$ is called convergent if there exists an $x_{0} \in X$ such that for every $\varepsilon>0$ and $\alpha \in A$, there is an $N$ with $p_{\alpha}\left(x_{0}, x_{n}\right) \leq \varepsilon$ for all $n \geq N$.

Definition 3. A gauge space is called sequentially complete if any Cauchy sequence is convergent.

A subset of $X$ is said to be sequentially closed if it contains the limit of any convergent sequence of its elements.

For further details see J. Dugundji [5], A. Granas, J. Dugundji [8].

Let $X$ be a nonempty set an $f: X \rightarrow X$ be an operator. Then $x \in X$ is called fixed point for $f$ if and only if $x=f(x)$. The set Fix $(f):=\{x \in X \mid x=f(x)\}$ is called the fixed point set of $f$.

Definition 4. Let $(X, \mathcal{P})$ be a gauge space and let $f:(X, \mathcal{P}) \rightarrow(X, \mathcal{P})$ be a single-valued operator. By definition, $f$ is weakly Picard (briefly WPO) operator if the sequence of successive approximations $f^{n}(x)$ converges for all $x \in X$ and the limit (which may depend on $\mathrm{X}$ ) is a fixed point of $f$.

If $f$ is WPO, then we consider the operator $f^{\infty}:(X,(P)) \rightarrow(X,(P))$ defined by $f^{\infty}(x)=\lim _{n \rightarrow \infty} f^{n}(x)$.

Definition 5. Let $(X, \mathcal{P})$ be a gauge space and let $f:(X, \mathcal{P}) \rightarrow(X, \mathcal{P})$ be a WPO and $\psi=\left\{\psi_{\alpha}\right\}_{\alpha \in A}$ be a family of mappings such that $\psi_{\alpha}: \mathbb{R}_{+} \rightarrow \mathbb{R}_{+}$increasing, continuous in 0 and $\psi_{\alpha}(0)=0$. By definition the operator $f$ is $\psi_{\alpha}$-WPO if

$$
p_{\alpha}\left(x, f^{\infty}(x)\right) \leq \psi_{\alpha}\left(p_{\alpha}(x, f(x))\right) \text {, for all } x \in X, \alpha \in A .
$$

If there exists $c=\left\{c_{\alpha}\right\}_{\alpha \in A} \in(0, \infty)^{A}$ such that $\psi_{\alpha}(t):=c_{\alpha} \cdot t$, for each $t \in \mathbb{R}_{+}$ and $\alpha \in A$ then the operator $f$ is $c_{\alpha}$-WPO.

For the theory of weakly Picard operators, see [11] for the single-valued case. 
The purpose of this paper is to present some results concerning the Hyers-Ulam stability of some operatorial inclusions (such as the fixed point inclusion, the coincdence point equation or inclusion, etc.) in gauge spaces, using the weakly Picard operator technique.

\section{HyERS-UlAM STABILITY FOR FIXED POINT EQUATIONS}

We will present first the concept of Hyers-Ulam stability in the setting of gauge spaces.

Definition 6. Let $(X, \mathcal{P})$ be a gauge space and let $f:(X, \mathcal{P}) \rightarrow(X, \mathcal{P})$ be a single-valued operator. The fixed point equation

$$
x=f(x), x \in X
$$

is called generalized Hyers-Ulam stable if and only if there exists $\psi=\left\{\psi_{\alpha}\right\}_{\alpha \in A}$ a family of mappings, $\psi_{\alpha}: \mathbb{R}_{+} \rightarrow \mathbb{R}_{+}$increasing, continuous in 0 and $\psi_{\alpha}(0)=0$ such that for each $\varepsilon=\left\{\varepsilon_{\alpha}\right\}_{\alpha \in A} \in(0, \infty)^{A}$ and for each solution $y^{*}$ of the inequation

$$
p_{\alpha}(y, f(y)) \leq \varepsilon_{\alpha}, \alpha \in A,
$$

there exists a solution $x^{*}$ of the fixed point equation (2.1) such that

$$
p_{\alpha}\left(y^{*}, x^{*}\right) \leq \psi_{\alpha}\left(\varepsilon_{\alpha}\right) \text {, for all } \alpha \in A .
$$

If there exists $c=\left\{c_{\alpha}\right\}_{\alpha \in A} \in(0, \infty)^{A}$ such that $\psi_{\alpha}(t):=c_{\alpha} \cdot t$, for each $t \in \mathbb{R}_{+}$ and $\alpha \in A$ then the fixed point equation (2.1) is said to be Hyers-Ulam stable.

We refer to [12] for the particular case of Hyers-Ulam stability in metric spaces. Our first abstract result is as follows.

Theorem 1. Let $(X, \mathcal{P})$ be a gauge space and let $f:(X, \mathcal{P}) \rightarrow(X, \mathcal{P})$ be a $\psi_{\alpha}$ WPO. Then, the fixed point equation (2.1) is generalized Hyers-Ulam stable.

Proof. Let $\varepsilon=\varepsilon_{\alpha} \in(0, \infty)^{A}$ and let $y^{*} \in f^{\infty}(x, y)$ be an $\varepsilon$-solution of (2.2), i.e., $p_{\alpha}\left(y^{*}, f\left(y^{*}\right)\right) \leq \varepsilon_{\alpha}$, for all $\alpha \in A$. Since $f$ is a $\psi_{\alpha}$-WPO, for each $x \in X$ and $\alpha \in A$ we have

$$
p_{\alpha}\left(x, f^{\infty}(x) \leq \psi_{\alpha}\left(p_{\alpha}(x, f(x))\right) .\right.
$$

Then choosing $x^{*}=f^{\infty}\left(y^{*}\right)$ we have

$$
p_{\alpha}\left(y^{*}, x^{*}\right)=p_{\alpha}\left(y^{*}, f^{\infty}\left(y^{*}\right)\right) \leq \psi_{\alpha}\left(p_{\alpha}\left(y^{*}, f\left(y^{*}\right)\right)\right) \leq \psi_{\alpha}\left(\varepsilon_{\alpha}\right) .
$$

Thus the fixed point equation (2.1) is generalized Hyers-Ulam stable.

In 1974, Tarafdar [13] expressed the notion of contraction in Hausdorff uniform spaces, using the observation that a uniformity on $X$ determines a family of gauges $\left\{p_{\alpha}\right\}$. A Hyers-Ulam stability result for the case of Tarafdar contraction in gauge spaces is as follows. 
Theorem 2. Let $(X, \mathcal{P})$ be a gauge space and let $f:(X, \mathcal{P}) \rightarrow(X, \mathcal{P})$ be an $a_{\alpha}$-contraction, i.e. for every $\alpha \in A$ there exists $a=\left\{a_{\alpha}\right\}_{\alpha \in A} \in(0,1)^{A}$ such that

$$
p_{\alpha}(f(x), f(y)) \leq a_{\alpha} \cdot p_{\alpha}(x, y), \text { for all } x, y \in X .
$$

Then $F_{f}=\left\{x^{*}\right\}$ and the fixed point equation (2.1) is Hyers-Ulam stable.

Proof. From Tarafdar [13] we get that $f$ has a unique fixed point $x^{*} \in X$ and, for each $x \in X$, we have that $f^{n}(x) \rightarrow x^{*}$. Thus, $f$ is a Picard operator. Moreover, it is a $c_{\alpha}$-WPO, with $c_{\alpha}:=\frac{1}{1-a_{\alpha}}$. Applying Theorem 1 we obtain the conclusion.

An extension of the previous result concerns the case of graphic-contractions.

Theorem 3. Let $(X, \mathcal{P})$ be a sequentially complete gauge space. Let $f:(X, \mathcal{P}) \rightarrow$ $(X, \mathcal{P})$ be an operator. If $f$ is a graphic $a_{\alpha}$-contraction, i.e., for every $\alpha \in A$ there exists $a=\left\{a_{\alpha}\right\}_{\alpha \in A} \in(0,1)^{A}$ such that

$$
p_{\alpha}\left(f^{2}(x), f(x)\right) \leq a_{\alpha} \cdot p_{\alpha}(x, f(x)), \text { for all } x \in X
$$

and $f$ has closed graph, then $F_{f} \neq \varnothing$ and the equation (2.1) is Hyers-Ulam stable.

Proof. Let $x_{0} \in X$ and $x_{n} \in f\left(x_{n-1}\right)=f^{n}\left(x_{0}\right), n=1,2, \ldots$ If $m$ and $n$ are positive integers, $m<n$, then for each $\alpha \in A$ we have:

$$
\begin{aligned}
p_{\alpha}\left(x_{m}, x_{n}\right)= & p_{\alpha}\left(f^{m}\left(x_{0}\right), f^{n}\left(x_{0}\right)\right) \\
\leq & p_{\alpha}\left(f^{m}\left(x_{0}\right), f^{m+1}\left(x_{0}\right)\right)+p_{\alpha}\left(f^{m+1}\left(x_{0}\right), f^{m+2}\left(x_{0}\right)\right)+\ldots \\
& +p_{\alpha}\left(f^{n-1}\left(x_{0}\right), f^{n}\left(x_{0}\right)\right) \\
\leq & a_{\alpha} p_{\alpha}\left(f^{m-1}\left(x_{0}\right), f^{m}\left(x_{0}\right)\right)+a_{\alpha} p_{\alpha}\left(f^{m}\left(x_{0}\right), f^{m+1}\left(x_{0}\right)\right)+\ldots \\
& +a_{\alpha} p_{\alpha}\left(f^{n-2}\left(x_{0}\right), f^{n-1}\left(x_{0}\right)\right) \\
\leq & a_{\alpha}^{m} p_{\alpha}\left(x_{0}, f\left(x_{0}\right)\right)+a_{\alpha}^{m+1} p_{\alpha}\left(x_{0}, f\left(x_{0}\right)\right)+\ldots+a_{\alpha}^{n-1} p_{\alpha}\left(x_{0}, f\left(x_{0}\right)\right) \\
= & p_{\alpha}\left(x_{0}, f\left(x_{0}\right)\right) a_{\alpha}^{m}\left(1+a_{\alpha}+\ldots+a_{\alpha}^{n-m+1}\right) \\
\leq & p_{\alpha}\left(x_{0}, f\left(x_{0}\right)\right) a_{\alpha}^{m} \frac{1-a_{\alpha}^{n-m}}{1-a_{\alpha}} .
\end{aligned}
$$

Hence the sequence $\left(x_{n}\right)$ is Cauchy, therefore $\left(x_{n}\right)$ converges to a point $x^{*} \in X$. From the continuity of $f$ we get that $x^{*}$ is a fixed point for $f$. So, we have

$$
p_{\alpha}\left(x_{m}, x_{n}\right) \leq p_{\alpha}\left(x_{0}, f\left(x_{0}\right)\right) a_{\alpha}^{m} \frac{1-a_{\alpha}^{n-m}}{1-a_{\alpha}} .
$$

If we choose in the above inequality $m=0$ and let $n \rightarrow \infty$ we obtain:

$$
p_{\alpha}\left(x_{0}, x^{*}\right) \leq p_{\alpha}\left(x_{0}, f\left(x_{0}\right)\right) \frac{1}{1-a_{\alpha}}, \text { for all } \alpha \in A .
$$

Thus $f$ is a $c_{\alpha}$-WPO with $c_{\alpha}:=\frac{1}{1-a_{\alpha}}$. Therefore the second conclusion follows from Theorem 1 . 


\section{ApPlications}

We will apply some of the above results to nonlinear integral equations on the real axis.

$$
x(t)=\int_{0}^{t} K(t, s, x(s)) d s+g(t), t \in \mathbb{R}_{+} .
$$

We give the notion of Hyers-Ulam stability for the integral equation.

Definition 7. The integral equation (3.1) is called Hyers-Ulam stable if and only if there exists $c=\left\{c_{\alpha}\right\}_{\alpha \in A} \in(0, \infty)^{A}$ such that for each $\varepsilon=\left\{\varepsilon_{\alpha}\right\}_{\alpha \in A} \in(0, \infty)^{A}$ and for any $\varepsilon$-solution $y^{*}$ of (1) (i.e., any $y^{*} \in C\left([0, \infty], \mathbb{R}^{n}\right)$ which satisfies the inequality

$$
\left.\left|y^{*}(t)-\int_{0}^{t} K(t, s, x(s)) d s-g(t)\right| \leq \varepsilon_{\alpha}, \text { for each } t \geq 0\right)
$$

there exists a solution $x^{*}$ of the equation (3.1) such that

$$
\left|y^{*}(t)-x^{*}(t)\right| \leq c_{\alpha} \cdot \varepsilon_{\alpha}, \text { for each } t \geq 0 .
$$

Theorem 4. Consider equation (3.1). Suppose that:

i) $K: \mathbb{R}_{+} \times \mathbb{R}_{+} \times \mathbb{R}^{n} \rightarrow \mathbb{R}^{n}$ and $g: \mathbb{R}_{+} \rightarrow \mathbb{R}^{n}$ are continuous;

ii) there exists $k>0$ such that

$$
|K(t, s, u)-K(t, s, v)| \leq k|u-v|, \text { for each } t, s \in \mathbb{R}_{+}, u, v \in \mathbb{R}^{n} ;
$$

Then the integral equation (3.1) has a unique solution $x^{*}$ in $C\left([0,+\infty), \mathbb{R}^{n}\right)$ and equation (3.1) is Hyers-Ulam stable.

Proof. Let $X:=C\left([0,+\infty), \mathbb{R}^{n}\right)$ and the family of pseudo-norms

$$
\|x\|_{n}:=\max _{t \in[0, n]}|x(t)| e^{-\tau t}, \text { where } \tau>0 .
$$

Define now $d_{n}(x, y):=\|x-y\|_{n}$ for $x, y \in X$.

Then $\mathcal{P}:=\left(d_{n}\right)_{n \in \mathbb{N}^{*}}$ is family of gauges on $X$. Then $(X, \mathcal{P})$ is a complete gauge space.

Define $A: C\left([0,+\infty), \mathbb{R}^{n}\right) \rightarrow C\left([0,+\infty), \mathbb{R}^{n}\right)$, by the formula

$$
A x(t):=\int_{0}^{t} K(t, s, x(s)) d s+g(t), t \in \mathbb{R}_{+} .
$$

For each $x, y \in X$ and for $t \in[0, n]$, we have successively:

$$
\begin{gathered}
|A x(t)-A y(t)| \leq \int_{0}^{t}|K(t, s, x(s))-K(t, s, y(s))| d s \leq \int_{0}^{t} k|x(s)-y(s)| d s \\
=k \int_{0}^{t}|x(s)-y(s)| e^{-\tau s} e^{\tau s} d s \leq k \int_{0}^{t} e^{\tau s}\left(|x(s)-y(s)| e^{-\tau s}\right) d s \\
\leq k d_{n}(x, y) \int_{0}^{t} e^{\tau s} d s \leq \frac{k}{\tau} d_{n}(x, y) e^{\tau t} .
\end{gathered}
$$


Hence, for $\tau>k$ and denoting $L:=\frac{k}{\tau}<1$ we obtain

$$
d_{n}(A x, A y) \leq L d_{n}(x, y) \text {, for each } x, y \in X .
$$

The conclusion follows now from Theorem 2 .

Consider now the following equation

$$
x(t)=\int_{-t}^{t} K(t, s, x(s)) d s+g(t), t \in \mathbb{R} .
$$

Theorem 5. Consider the equation (3.3). Suppose that:

i) $K: \mathbb{R} \times \mathbb{R} \times \mathbb{R}^{n} \rightarrow \mathbb{R}^{n}$ and $g: \mathbb{R} \rightarrow \mathbb{R}^{n}$ are continuous;

ii) there exists $k>0$ such that

$$
|K(t, s, u)-K(t, s, v)| \leq k|u-v|, \text { for each } t, s \in \mathbb{R}, u, v \in \mathbb{R}^{n} ;
$$

Then the integral equation (3.3) has a unique solution $x^{*}$ in $C\left(\mathbb{R}, \mathbb{R}^{n}\right)$ and equation (3.3) is Hyers-Ulam stable.

Proof. We consider the gauge space $X:=\left(C\left(\mathbb{R}, \mathbb{R}^{n}\right), \mathcal{P}:=\left(d_{n}\right)_{n \in \mathbb{N}}\right)$ where

$$
d_{n}(x, y)=\max _{-n \leq t \leq n}\left(|x(t)-y(t)| \cdot e^{-\tau|t|}\right), \tau>0,
$$

and the operator $B: X \rightarrow X$ defined by

$$
B x(t)=\int_{-t}^{t} K(t, s, x(s)) d s+g(t) .
$$

From condition (ii), for $x, y \in X$, we have

$$
\begin{gathered}
|B x(t)-B y(t)| \leq \int_{-t}^{t} k|x(s)-y(s)| e^{-\tau|s|} e^{\tau|s|} d s \leq \\
k \int_{-t}^{t} e^{\tau|s|}\left(|x(s)-y(s)| e^{-\tau|s|}\right) d s \leq k d_{n}(x, y)\left|\int_{-t}^{t} e^{\tau|s|} d s\right| \leq \\
k d_{n}(x, y) \int_{-|t|}^{|t|} e^{\tau|s|} d s \leq \frac{2 k}{\tau} d_{n}(x, y) e^{\tau|t|}, t \in[-n ; n] .
\end{gathered}
$$

Thus, for any $\tau \geq 2 k$, if we denote $L:=\frac{2 k}{\tau}<1$, we obtain

$$
\left.d_{n}(B(x), B(y)) \leq L d_{n}(x, y)\right) \text {, for all } x, y \in E \text {, and for } n \in \mathbb{N} \text {. }
$$

The conclusion follows again by Theorem 2 .

\section{ACKNOWLEDGEMENT}

For the first author, this work was possible with the financial support of a grant of the Romanian National Authority for Scientific Research, CNCS-UEFISCDI, project number PN-II-ID-PCE-2011-3-0094. 


\section{REFERENCES}

[1] V. G. Angelov, "Fixed point theorem in uniform spaces and applications," Czech. Math. J., vol. 37(112), no. 1, pp. 19-33, 1987.

[2] V. G. Angelov, "Fixed points of multi-valued mappings in uniform spaces," Math. Balk., New Ser., vol. 12, no. 1-2, pp. 29-35, 1998.

[3] G. L. j. Cain and M. Z. Nashed, "Fixed points and stability for a sum of two operators in locally convex spaces," Pac. J. Math., vol. 39, pp. 581-592, 1971.

[4] I. Colojoară, "Sur un théorème d'un point fixe dans les espaces uniformes complets," Comun. Acad. Republ. Popul. Romine, vol. 11, pp. 281-283, 1961.

[5] J. Dugundji, Topology, ser. Advanced Mathematics. Boston: Allyn and Bacon, Inc., 1966.

[6] M. Frigon, "Fixed point results for generalized contractions in gauge spaces and applications," Proc. Am. Math. Soc., vol. 128, no. 10, pp. 2957-2965, 2000.

[7] N. Gheorghiu, "Ein satz über Kontraktionen in uniformen Räumen," Stud. Cercet. Mat., vol. 19, pp. 119-122, 1967.

[8] A. Granas and J. Dugundji, Fixed point theory, ser. Springer Monographs in Mathematics. New York: Springer, 2003.

[9] R. J. Knill, "Fixed points of uniform contractions," J. Math. Anal. Appl., vol. 12, pp. 449-455, 1965.

[10] G. Marinescu, Topologische und pseudotopologische Vektorräume [Spaţii vectoriale topologice si pseudotopologice], ser. Biblioteca Matematică. Bucureşti: Editura Academiei Republicii Populare Romine, 1959, vol. 4.

[11] I. A. Rus, "Picard operators and applications," Sci. Math. Jpn., vol. 58, no. 1, pp. 191-219, 2003.

[12] I. A. Rus, "Remarks on ulam stability of the operatorial equations," Fixed Point Theory, vol. 10, no. 2, pp. 305-320, 2009.

[13] E. Tarafdar, "An approach to fixed-point theorems on uniform spaces," Trans. Am. Math. Soc., vol. 191, pp. 209-225, 1974.

\section{Authors' addresses}

\section{Bota}

Babeş-Bolyai University, Department of Applied Mathematics, Kogalniceanu Str. No 1., ClujNapoca, Romania

E-mail address: bmonica@math.ubbcluj.ro

\section{T. P. Petru}

Babeş-Bolyai University, Faculty of Economics and Business Administration, Teodor Mihali Str. No 1., Cluj-Napoca, Romania

E-mail address: petra.petrudecon.ubbcluj.ro

\section{G. Petruşel}

Babeş-Bolyai University, Faculty of Business, Horea Str. No 7., Cluj-Napoca, Romania

E-mail address: gabi.petrusel@tbs.ubbcluj.ro 